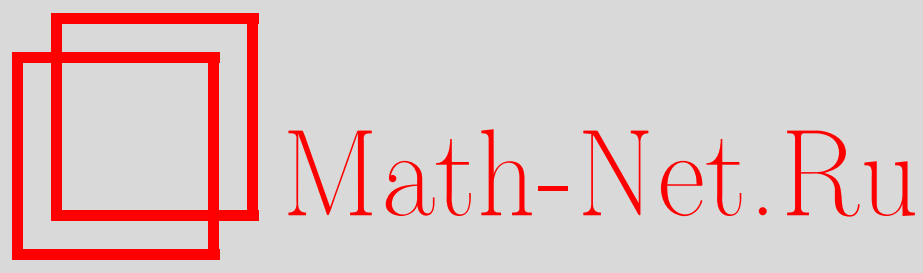

В. Е. Зобов, Квантовые и классические корреляции в высокотемпературной динамике двух связанных больших спинов, ТМФ, 2013, том 177, номер 1, 111-125

DOI: https://doi.org/10.4213/tmf8510

Использование Общероссийского математического портала Math-Net.Ru подразумевает, что вы прочитали и согласны с пользовательским соглашением http://www.mathnet.ru/rus/agreement

Параметры загрузки:

IP: 18.234 .156 .22

26 апреля 2023 г., 11:53:05

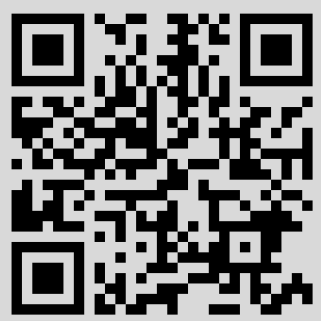




\title{
КВАНТОВЫЕ И КЛАССИЧЕСКИЕ КОРРЕЛЯЦИИ В ВЫСОКОТЕМПЕРАТУРНОЙ ДИНАМИКЕ ДВУХ СВЯЗАННЫХ БОЛЬШИХ СПИНОВ
}

\begin{abstract}
Исследуются динамические корреляции двух связанных спинов в зависимости от времени и величин спиновых квантовых чисел. В высокотемпературном приближении получены аналитические выражения для взаимной информации, квантовой и классической частей корреляций. Наряду с ортогональными измерениями рассмотрены неортогональные измерения с базисом из спиновых когерентных состояний. Показано, что на малых временах при увеличении спиновых квантовых чисел квантовая часть корреляций становится много меньше классической части, тогда как на временах, равных половине квантового периода, это не так.
\end{abstract}

Ключевые слова: спиновая динамика, высокотемпературное приближение, взаимная информация, обобщенные измерения, спиновые когерентные состояния, квантовые корреляции.

DOI: $1010.4231 / \operatorname{tmf} 8510$

\section{1. ВВЕДЕНИЕ}

K настоящему времени выполнено много экспериментальных работ, в которых методом ядерного магнитного резонанса (ЯМР) демонстрируется реализация квантовых алгоритмов на ядерных спинах при высоких температурах (см. обзор [1]). Известно, что квантовая запутанность отсутствует, но динамические корреляции, образующиеся в процессе квантовых вычислений, наряду с классической частью содержат квантовую часть [2], [3]. Причем эти высокотемпературные квантовые корреляции способны обеспечить быстрое выполнение некоторых квантовых алгоритмов [1]-[7]. Механизмы такого ускорения пока еще не выяснены, хотя анализу соотношения квантовых и классических корреляций посвящено много работ (см., например, обзор [7]).

Вместо произвольных состояний квантовых систем, для которых пока не получено общего решения для соотношения квантовых и классических частей корреляций [7], мы рассмотрим корреляции, типичные для ЯМР [8]. Для ядерных спинов

* Институт физики им. Л. В. Киренского СО РАН, Красноярск, Россия.

E-mail: rsa@iph.krasn.ru 
в сильном постоянном магнитном поле при комнатной температуре $T$ поляризация очень мала: $\beta=\hbar \omega_{0} / k T \approx 10^{-5} \ll 1$ ( $\omega_{0}-$ ларморовская частота), поэтому равновесную матрицу плотности при описании ЯМР берут в следующем виде:

$$
\hat{\rho}_{\mathrm{eq}}=\frac{1+\beta \widehat{S}_{z}}{Z}
$$

где $Z$ - статсумма, $\widehat{S}_{\alpha}=\sum_{j} \widehat{S}_{j \alpha}, \widehat{S}_{j \alpha}-\alpha$-компонента оператора спина $j, \alpha=x, y, z$, магнитное поле направлено по оси $z$. Для наблюдения сигнала ЯМР на систему действуют импульсом радиочастотного (РЧ) магнитного поля, вызывающим поворот спинов на угол $90^{\circ}$ вокруг оси у вращающейся системы координат $(\mathrm{BCK})$,

$$
\hat{\rho}(0)=\widehat{Y} \hat{\rho}_{\mathrm{eq}} \widehat{Y}^{-1}=\frac{1+\beta \widehat{S}_{x}}{Z} .
$$

Эта начальная матрица плотности будет изменяться во времени:

$$
\hat{\rho}(t)=\widehat{U}(t) \hat{\rho}(0) \widehat{U}^{-1}(t)=\frac{1+\beta \widehat{U}(t) \widehat{S}_{x} \widehat{U}^{-1}(t)}{Z},
$$

где $\widehat{U}(t)=e^{-i \widehat{H} t / \hbar}$. Поляризация отдельных спинов в поле (начальное упорядочение зеемановской подсистемы (1)) с течением времени переходит в корреляцию между спином и локальным полем на нем, созданным диполь-дипольными взаимодействиями. На малых временах она описывается в выражении (2) членом вида

$$
-\frac{i \beta t}{\hbar Z}\left[\widehat{H}_{\mathrm{d}}, \widehat{S}_{x}\right]=-\frac{i \beta t}{Z} \sum_{i<j} a_{i j}\left[\left(3 \widehat{S}_{i z} \widehat{S}_{j z}-\widehat{\vec{S}}_{i} \cdot \widehat{\vec{S}}_{j}\right), \widehat{S}_{x}\right]=\frac{3 \beta t}{Z} \sum_{i \neq j} a_{i j} \widehat{S}_{i z} \widehat{S}_{j y}
$$

Эта корреляция хорошо изучена для ЯМР, поскольку ответственна за солид-эхо [9], если подействовать на систему вторым РЧ-импульсом поворота на $90^{\circ}$ вокруг оси $x$ BCK. Если второй РЧ-импульс повернет спины только на угол $45^{\circ}$, то указанная корреляция превращается в упорядочение спин-спиновой подсистемы, обеспечивающее отличное от нуля среднее значение энергии диполь-дипольных взаимодействий [10]. Однако разделение таких корреляций (3) на квантовую и классическую части не проводилось.

В настоящей работе мы выполним такое разделение для модельной системы. Возьмем систему двух больших спинов $S_{1}$ и $S_{2}$ с диполь-дипольным взаимодействием между проекциями на ось $z$, заданным гамильтонианом

$$
\widehat{H}_{S S}=\hbar \frac{J}{S_{2}} \widehat{S}_{1 z} \widehat{S}_{2 z}
$$

Для исследования предела $S_{1}, S_{2} \rightarrow \infty$ мы, следуя работам [11], [12], взяли константу взаимодействия, поделенную на величину спина $S_{2} \geqslant S_{1}$. Такая модель позволяет проследить переход от случая двух спинов $S_{2}=S_{1}=1 / 2$, где динамические корреляции делятся на квантовую и классическую части поровну [13], к случаю двух классических магнитных моментов, в котором останется только одна часть. Большой спин в ядерных системах образуется, например, в случае межмолекулярного взаимодействия быстровращающихся симметричных молекул адамантана в твердой 
матрице. Из-за быстрого вращения выравниваются по величине константы дипольного взаимодействия 16 ядер ${ }^{1} \mathrm{H}$ одной молекулы с ядром ${ }^{13} \mathrm{C}$ другой молекулы [14]. Еще большее число ядер с равными константами взаимодействия может быть получено для подвижных молекул в нанополостях [15].

Исследование спинов $S>1 / 2$ представляется важным не только для теории ЯМР, но и для теории квантовых компьютеров, поскольку квантовые вычисления могут быть выполнены на кудитах (квантовых системах с $d$ уровнями, для спинов $d=2 S+1)$. Использование кудитов обещает ряд преимуществ (например, на них можно получить тот же размер вычислительного базиса при меньшем числе спинов $\left.2^{n} \leftrightarrow d^{n}\right)$ [16]-[18], но такие системы недостаточно изучены. Опубликовано много работ, в которых проведены расчеты таких мер квантовых корреляций, как квантовый и геометрический дискорды для двух кубитов (двух спинов $S=1 / 2$ ) [7], [13], [19] или в случаях, сводящихся к ним [20]-[22]. Выполнен расчет этих мер для некоторых состояний двух спинов $S=1$ [23] и симметричных состояний с большим $d$ [24]. Дана оценка их значений для систем $2 \times n$ [3], [5], [25], [26], а также оценка геометрического дискорда для систем $m \times n$ [26]-[28].

Динамика корреляций двух связанных больших спинов до сих пор не исследовалась, в настоящей работе она рассматривается в высокотемпературном приближении. Рассчитана эволюция во времени взаимной информации матрицы плотности и взаимной информации классической функции распределения по направлениям, полученной после обобщенных измерений (POVM-измерения) $)^{1)}$ [7], [29] с базисом из спиновых когерентных состояний (СКC) [22], [30], [31]. Исследуется зависимость соотношения квантовых и классических корреляций от величин спинов. Выполнено сравнение результатов, полученных с помощью ортогональных и неортогональных измерений.

\section{2. ВЗАИМНАЯ ИНФОРМАЦИЯ ДВУХ СПИНОВ}

Рассмотрим систему двух спинов $S_{1}$ и $S_{2}$ со спин-спиновым взаимодействием (4). Для каждого спина будем использовать базис из состояний с определенным значением проекции на ось $z:|m\rangle$, где $m$ принимает $2 S+1$ значений:

$$
-S, \quad-S+1, \quad \ldots, \quad S-1, \quad S .
$$

Для двух спинов будем использовать базис в виде прямого произведения $\left|m_{1}\right\rangle \otimes\left|m_{2}\right\rangle$.

Полагаем, что система находится в равновесии в сильном постоянном магнитном поле, превосходящем спин-спиновое взаимодействие (4), с матрицей плотности (1). Эволюция во времени матрицы (2) может быть выписана в явном виде:

$$
\begin{aligned}
\hat{\rho}(t)=e^{-i \widehat{H}_{S S} t / \hbar} \hat{\rho}(0) e^{i \widehat{H}_{S S} t / \hbar}=\frac{1}{Z}\left[1+\frac{\beta}{2}\left(\widehat{S}_{1+} e^{-i \tau \widehat{S}_{2 z}}+\right.\right. \\
\left.\left.\quad+\widehat{S}_{1-} e^{i \tau \widehat{S}_{2 z}}+\widehat{S}_{2+} e^{-i \tau \widehat{S}_{1 z}}+\widehat{S}_{2-} e^{i \tau \widehat{S}_{1 z}}\right)\right]=\frac{1}{Z}[1+\beta \Delta \hat{\rho}(t)],
\end{aligned}
$$

где $\widehat{S}_{j \pm}=\widehat{S}_{j x} \pm i \widehat{S}_{j y}, \tau=t J / S_{2}-$ безразмерное время, $Z=d_{1} d_{2}, d_{j}=2 S_{j}+1$.

\footnotetext{
1) Positive operator-valued measure.
} 


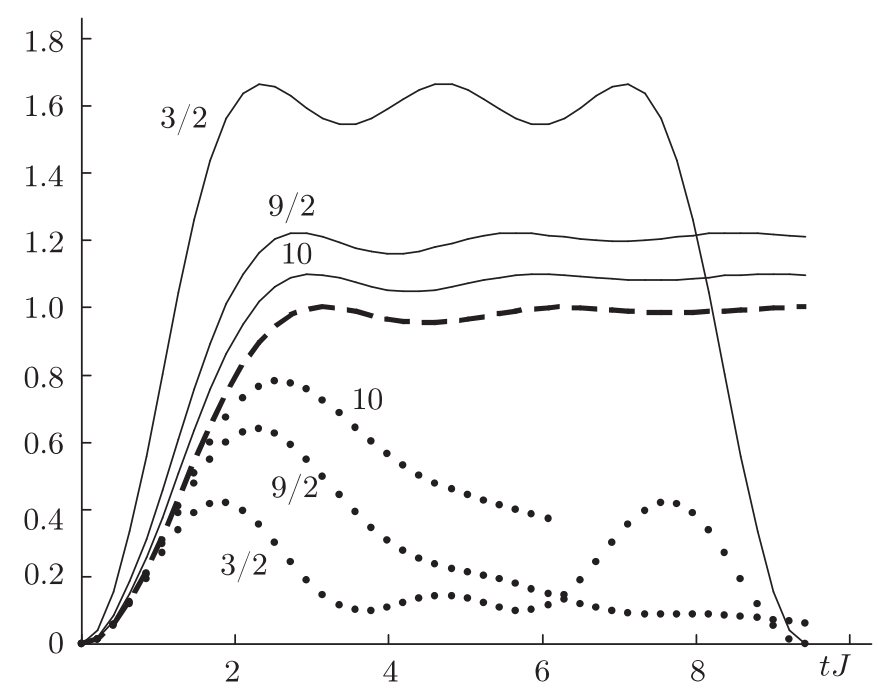

Рис. 1. Эволюция во времени взаимных информаций $\left(I(\hat{\rho}) /\left(2 b S^{2}\right)-\right.$ сплошные линии, $J_{\mathrm{c}}\left(P_{\mathrm{c}}\right) /\left(2 b S^{2}\right)$ - штриховая линия, $J_{\mathrm{BB}}\left(P_{12}\right) /\left(2 b S^{2}\right)$ - пунктирные линии) при разных значениях $S=S_{1}=S_{2}$, указанных числами у кривых.

Мерой корреляции между спинами служит взаимная информация [7], [29]

$$
I(\hat{\rho})=S_{\mathrm{N}}\left(\hat{\rho}_{1}\right)+S_{\mathrm{N}}\left(\hat{\rho}_{2}\right)-S_{\mathrm{N}}(\hat{\rho}),
$$

где $S_{\mathrm{N}}(\hat{\rho})=-\operatorname{Tr}\left\{\hat{\rho} \log _{2} \hat{\rho}\right\}-$ энтропия фон Неймана, $\hat{\rho}_{1}=\operatorname{Tr}_{2} \hat{\rho}, \hat{\rho}_{2}=\operatorname{Tr}_{1} \hat{\rho}-$ редуцированные матрицы плотности после вычисления следа матрицы (6) по состояниям второго или первого спина соответственно. Вычислив след, находим

$$
\begin{gathered}
\hat{\rho}_{1}=\frac{1+\beta \widehat{S}_{1 x} g_{2}(t)}{d_{1}}, \quad \hat{\rho}_{2}=\frac{1+\beta \widehat{S}_{2 x} g_{1}(t)}{d_{2}}, \\
g_{j}(t)=\frac{1}{d_{j}} \sum_{m=-S_{j}}^{S_{j}} e^{ \pm i \tau m}=\frac{\sin \left(d_{j} \tau / 2\right)}{d_{j} \sin (\tau / 2)} .
\end{gathered}
$$

Энтропию фон Неймана вычисляем в низшем порядке по $\beta$ [2], [3], [8]:

$$
S_{\mathrm{N}}(\hat{\rho})=-\operatorname{Tr}\left(\hat{\rho} \log _{2} \hat{\rho}\right) \approx \log _{2} Z-\frac{\beta^{2}}{2 Z \ln 2} \operatorname{Tr}(\Delta \hat{\rho})^{2}
$$

Находим

$$
I(\hat{\rho})=S_{1}\left(S_{1}+1\right) b\left[1-g_{2}^{2}(t)\right]+S_{2}\left(S_{2}+1\right) b\left[1-g_{1}^{2}(t)\right]
$$

где $b=\beta^{2} / 6 \ln 2$.

На рис. 1 показаны зависимости от времени полученных результатов для взаимной информации двух спинов (11) при разных значениях спинового квантового числа $S_{1}=S_{2}=S$ и классических моментов (см. в приложении формулу (П.5)). 
Время взято в безразмерных единицах $\tau S=t J$. Результаты приведены в единицах $2 b S^{2}$ (т. е. приведены отношения $\left.I(\hat{\rho}) / 2 b S^{2}\right)$. В таких единицах кривые зависимостей классической взаимной информации $J_{\mathrm{c}}\left(P_{\mathrm{c}}\right) / 2 b S^{2}(\Pi .5)$ для разных $S$ совпадают. При $t=0$ корреляций нет. При $\tau=t J / S \ll 1$ получаем

$$
\begin{aligned}
I(\hat{\rho}) \approx \frac{2}{3} b S_{1}\left(S_{1}+1\right) S_{2}\left(S_{2}+1\right) \tau^{2} & =\frac{2}{3} b S_{1}\left(S_{1}+1\right)\left(1+\frac{1}{S_{2}}\right)(J t)^{2}, \\
J_{\mathrm{c}}\left(P_{\mathrm{c}}\right) \approx \frac{2}{3} b\left(S_{1}\right)^{2}\left(S_{2}\right)^{2} \tau^{2} & =\frac{2}{3} b\left(S_{1}\right)^{2}(J t)^{2} .
\end{aligned}
$$

Квантовая взаимная информация (12) превосходит классическую (13), поскольку для нее вследствие квантовых флуктуаций квадрат полного момента равен $S_{j}\left(S_{j}+1\right)$, а не $S_{j}^{2}$.

На больших временах классическая взаимная информация $J_{\mathrm{c}}\left(P_{\mathrm{c}}\right)(\Pi .5)$ стремится к предельному значению. Квантовая взаимная информация $I(\hat{\rho})(11)$ имеет другое поведение: сначала выходит на плато, но затем снижается до нуля при $\tau=2 \pi$. Периодическое изменение во времени с периодом $T=2 \pi S / J$ обусловлено дискретностью уровней энергии (такое поведение простых квантовых систем хорошо известно, см., например, [11]-[13], [21]). При $t=T$ разница набега фаз разных энергетических уровней $(6)$ кратна $2 \pi$, поэтому $g_{j}^{2}(T)=1(9)$ и $I(\hat{\rho})=0$. В случае классических магнитных моментов их проекции на магнитное поле и энергии меняются непрерывно, поэтому $g_{\mathrm{c} j}(t) \rightarrow 0$ при $t \rightarrow \infty(\Pi .4)$ и кривая $J_{\mathrm{c}}\left(P_{\mathrm{c}}\right)(\Pi .5)$ выходит на плато. Для $S=3 / 2$ периодичность величины взаимной информации видна на рис. $1(I(\hat{\rho})=0$ при $t J=3 \pi)$. Для $S=9 / 2$ обращение взаимной информации в нуль произойдет за пределами показанной области при $t J=9 \pi$. При $S \rightarrow \infty$ период становится бесконечным, а на конечных временах функции $I(\hat{\rho}) / 2 b S^{2}(11)$ и $J_{\mathrm{c}}\left(P_{\mathrm{c}}\right) / 2 b S^{2}(\Pi .5)$ совпадают.

\section{3. ОРТОГОНАЛЬНЫЕ ИЗМЕРЕНИЯ}

Взаимная информация (7) служит мерой полных корреляций, являющихся суммой классических и квантовых корреляций. Для вычисления классических корреляций следует произвести измерение [7]. При измерении фон Неймана производится проецирование состояния $\hat{\rho}(t)$ на некоторый полный базис из ортогональных волновых функций $\left|\Psi_{m}\right\rangle$ с помощью полной системы проекторов:

$$
\widehat{\Pi}_{m}=\left|\Psi_{m}\right\rangle\left\langle\Psi_{m}\right|, \quad \sum_{m} \widehat{\Pi}_{m}=1 .
$$

В случае системы с $S_{1}=1 / 2$ полный набор взаимно ортогональных проекторов состоит из двух проекторов общего вида

$$
\widehat{\Pi}_{1 \pm}=\frac{1}{2}\left[1 \pm\left(n_{x} \hat{\sigma}_{1 x}+n_{y} \hat{\sigma}_{1 y}+n_{z} \hat{\sigma}_{1 z}\right)\right]
$$

где $n_{\alpha}$ - направляющие косинусы, $\hat{\sigma}_{\alpha}$ - матрицы Паули, $\alpha=x, y, z$. При обобщенных POVM-измерениях, которые мы рассмотрим в следующем разделе, функции $\left|\Psi_{m}\right\rangle$ в операторах (14) могут быть неортогональны, тогда эти операторы, строго говоря, уже не являются проекторами [29]. 
После проецирования по первому спину матрица плотности (6) преобразуется к виду

$$
\widehat{\Pi}_{1}(\hat{\rho})=\frac{1}{Z}\left[1+\beta \widehat{\Pi}_{1}(\Delta \hat{\rho}(t))\right]
$$

где

$$
\widehat{\Pi}_{1}(\Delta \hat{\rho}(t))=\sum_{m}\left(\widehat{\Pi}_{1 m} \otimes \widehat{E}_{2}\right) \Delta \hat{\rho}(t)\left(\widehat{\Pi}_{1 m} \otimes \widehat{E}_{2}\right)
$$

$\widehat{E}_{2}$ - единичная матрица. В низшем порядке по обратной температуре находим для взаимной информации (7)

$$
\begin{aligned}
I\left(\widehat{\Pi}_{1}(\hat{\rho})\right) & =S_{\mathrm{N}}\left(\widehat{\Pi}_{1}\left(\hat{\rho}_{1}\right)\right)+S_{\mathrm{N}}\left(\widehat{\Pi}_{1}\left(\hat{\rho}_{2}\right)\right)-S_{\mathrm{N}}\left(\widehat{\Pi}_{1}(\hat{\rho})\right)= \\
& =\frac{\beta^{2}}{2 \ln 2}\left\{\frac{1}{Z} \operatorname{Tr}\left(\widehat{\Pi}_{1}(\Delta \hat{\rho})\right)^{2}-\frac{1}{d_{1}} \operatorname{Tr}_{1}\left(\widehat{\Pi}_{1}\left(\Delta \hat{\rho}_{1}\right)\right)^{2}-\frac{1}{d_{2}} \operatorname{Tr}_{2}\left(\Delta \hat{\rho}_{2}\right)^{2}\right\} .
\end{aligned}
$$

Величина этой меры классической корреляции зависит от выбранного базиса (14). Предлагается [7] перебрать все базисы и в качестве универсальной меры взять максимальное значение корреляции (17). Такую программу удается выполнить только в некоторых простых случаях, например для двухуровневой системы, которую мы рассмотрим ниже.

Если вычесть из всех корреляций (7) классическую часть (17), мы получим квантовую часть корреляций:

$$
\begin{aligned}
Q & =I(\hat{\rho})-I\left(\widehat{\Pi}_{1}(\hat{\rho})\right)= \\
& =\frac{\beta^{2}}{2 \ln 2}\left\{\frac{1}{Z} \operatorname{Tr}(\Delta \hat{\rho})^{2}-\frac{1}{Z} \operatorname{Tr}\left(\widehat{\Pi}_{1}(\Delta \hat{\rho})\right)^{2}-\frac{1}{d_{1}} \operatorname{Tr}_{1}\left(\Delta \hat{\rho}_{1}\right)^{2}+\frac{1}{d_{1}} \operatorname{Tr}_{1}\left(\widehat{\Pi}_{1}\left(\Delta \hat{\rho}_{1}\right)\right)^{2}\right\} .
\end{aligned}
$$

После проведения минимизации этой величины по измерительным базисам получаем энтропийную меру квантовых корреляций - квантовый дискорд [7]. Мера (18) без проведения оптимизации получила название дискорд, зависящий от измерительного базиса [7].

Для упрощения вычислений вместо рассмотренной выше энтропийной меры была предложена геометрическая мера квантовых корреляций - геометрический дискорд [7], для которого в высокотемпературном приближении находим

$$
D_{\mathrm{G}}=\min _{\Pi_{1}} \operatorname{Tr}\left(\hat{\rho}-\widehat{\Pi}_{1}(\hat{\rho})\right)^{2}=\frac{\beta^{2}}{Z^{2}} \min _{\Pi_{1}}\left\{\operatorname{Tr}(\Delta \hat{\rho})^{2}-\operatorname{Tr}\left(\widehat{\Pi}_{1}(\Delta \hat{\rho})\right)^{2}\right\} .
$$

При выводе использовано свойство $\operatorname{Tr}(\widehat{\Pi}(\Delta \hat{\rho}))^{2}=\operatorname{Tr}(\widehat{\Pi}(\Delta \hat{\rho}) \Delta \hat{\rho})$. Несмотря на имеющиеся у этой меры недостатки, отмеченные в работах [28], [32], [33], она в настоящее время часто используется для оценки квантовых корреляций [3], [19]-[28]. Для улучшения этой меры в работе [28] предложено изменить ее следующим образом:

$$
\widetilde{D}_{\mathrm{G}}=\frac{D_{\mathrm{G}}}{\operatorname{Tr}(\hat{\rho})^{2}} .
$$

В высокотемпературном приближении этому преобразованию соответствует умножение (19) на $Z$. 
Выполним расчет указанных мер для двухспиновой системы (4) в состоянии (6) при $S_{1}=1 / 2, S_{2} \geqslant 1 / 2$. Применив проекторы (15) и выполнив вычисления, находим

$$
\begin{aligned}
& \frac{\operatorname{Tr}\left(\widehat{\Pi}_{1}(\Delta \hat{\rho})\right)^{2}}{Z}= \\
& \quad=\frac{S_{2}\left(S_{2}+1\right)}{3}\left(\cos ^{2} \frac{\tau}{2}+n_{z}^{2} \sin ^{2} \frac{\tau}{2}\right)+\frac{n_{x}^{2}}{8}\left(1+g_{2}(2 t)\right)+\frac{n_{y}^{2}}{8}\left(1-g_{2}(2 t)\right), \\
& \frac{I\left(\widehat{\Pi}_{1}(\hat{\rho})\right)}{3 b}= \\
& \quad=\frac{S_{2}\left(S_{2}+1\right)}{3} n_{z}^{2} \sin ^{2} \frac{\tau}{2}+\frac{n_{x}^{2}}{8}\left(1+g_{2}(2 t)\right)+\frac{n_{y}^{2}}{8}\left(1-g_{2}(2 t)\right)-\frac{n_{x}^{2}}{4} g_{2}^{2}(t) .
\end{aligned}
$$

Максимальное значение величины (22) достигается при направляющих косинусах $n_{x}=n_{y}=0, n_{z}=1$ для любых значений времени. Отсюда находим для классических корреляций и квантового дискорда соответственно

$$
C_{\mathrm{N}}=\max _{\widehat{\Pi}_{1}} I\left(\widehat{\Pi}_{1}(\hat{\rho})\right)=S_{2}\left(S_{2}+1\right) b \sin ^{2} \frac{\tau}{2}, \quad D_{\mathrm{N}}=I(\hat{\rho})-C_{\mathrm{N}}=\frac{3}{4} b\left[1-g_{2}^{2}(t)\right] .
$$

Для величины (21) максимум достигается при $n_{x}=1$ на малых временах $\tau<\tau_{\mathrm{c}}$, а на больших временах $\tau>\tau_{\mathrm{c}}$ при $n_{z}=1$. Здесь $\tau_{\mathrm{c}}-$ решение уравнения

$$
\frac{S_{2}\left(S_{2}+1\right)}{3} \sin ^{2} \frac{\tau}{2}=\frac{1+g_{2}(2 t)}{8} .
$$

При $d_{2}=2$ находим $\tau_{\mathrm{c}}=\pi / 2$, при $d_{2}=3-\tau_{\mathrm{c}}=0.574$. Наконец, при $d_{2} \gg 1$ получаем оценку $\tau_{\mathrm{c}} \approx \sqrt{3} / \sqrt{2 S_{2}\left(S_{2}+1\right)} \approx \sqrt{6} / d_{2}$. В итоге для геометрического дискорда находим

$$
D_{\mathrm{G}}= \begin{cases}\frac{\beta^{2}}{Z}\left[\frac{S_{2}\left(S_{2}+1\right)}{3} \sin ^{2} \frac{\tau}{2}+\frac{1-g_{2}(2 t)}{8}\right], & \tau<\tau_{\mathrm{c}} \\ \frac{\beta^{2}}{4 Z}, & \tau>\tau_{\mathrm{c}}\end{cases}
$$

Временно́е поведение двух мер схоже. Корреляции растут и выходят на плато. Различия заключаются в способе выхода на плато и в высоте плато. У энтропийной меры эта высота не зависит от $d_{2}$, тогда как у геометрической меры она уменьшается как $1 / d_{2}$ из-за наличия дополнительного множителя $1 / Z$ от $\hat{\rho}^{2}$ в определении (19) по сравнению с $\hat{\rho} \log _{2} \hat{\rho}$ в определении (18). Такие различия отмечены в работах [3], [28] для кубита $\left(d_{1}=2\right)$, связанного с многокубитовой системой $\left(d_{2}=2^{n}\right)$ по схеме детерминистического квантового вычисления с одним кубитом (DQC1). Они устраняются преобразованием (20).

Для двухспиновой системы с произвольными спинами будем проецировать состояние (6) на собственные состояния (5) оператора $S_{1 z}$ с проекторами

$$
\widehat{\Pi}_{S 1 m}=|m\rangle\langle m| .
$$

В этом случае для классических корреляций и дискорда, зависящего от измерительного базиса, находим соответственно

$$
C_{\mathrm{N}}^{(S 1)}=I\left(\widehat{\Pi}_{S 1}(\hat{\rho})\right)=S_{2}\left(S_{2}+1\right) b\left[1-g_{1}^{2}(t)\right], \quad Q_{\mathrm{N}}^{(S 1)}=S_{1}\left(S_{1}+1\right) b\left[1-g_{2}^{2}(t)\right] .
$$




\section{4. НЕОРТОГОНАЛЬНЫЕ ИЗМЕРЕНИЯ}

Согласно полученному в предыдущем разделе результату (26) на малых временах $C_{\mathrm{N}}^{(S 1)}=Q_{\mathrm{N}}^{(S 1)}$ (при $S_{1}=S_{2}$ это соотношение сохраняется на всех временах). Таким образом, для ортогонального измерения с выбранным базисом мы получили, что квантовые корреляции могут сохраняться у больших спинов $S_{2} \rightarrow \infty$. Сохранение квантовых свойств в этом пределе, проявляющееся в нарушении неравенств Белла, для синглетного состояния двух спинов отмечено в работах [34], [35]. Для систем в других состояниях ожидается переход при $S \rightarrow \infty$ от квантового спина к классическому моменту [11], [31], [36]. Уточним, к какому случаю относится наше состояние (6). С этой целью поменяем измерительный базис.

Считается, что наиболее близким к состояниям классического момента являются СКC (состояния Блоха) [30]:

$$
|\theta, \varphi\rangle=R(\theta, \varphi)|S\rangle=\sum_{m=-S}^{S}\left(\begin{array}{c}
2 S \\
S+m
\end{array}\right)^{1 / 2}\left(\cos \frac{\theta}{2}\right)^{S+m}\left(e^{i \varphi} \sin \frac{\theta}{2}\right)^{S-m}|m\rangle,
$$

где $\theta$ и $\varphi$ - соответственно полярный и азимутальный углы на единичной сфере (сфере Блоха). Эти состояния получаются из основного состояния $|S\rangle$ с помощью оператора поворота $R(\theta, \varphi)$ и являются суперпозицией состояний (5) с разными проекциями $m$. В состоянии (27) средние значения проекций спина

$$
\begin{gathered}
\left\langle\theta, \varphi\left|\widehat{S}_{z}\right| \theta, \varphi\right\rangle=S \cos \theta, \quad\left\langle\theta, \varphi\left|\widehat{S}_{x}\right| \theta, \varphi\right\rangle=S \sin \theta \cos \varphi \\
\left\langle\theta, \varphi\left|\widehat{S}_{y}\right| \theta, \varphi\right\rangle=S \sin \theta \sin \varphi
\end{gathered}
$$

те же, что и у классического момента (П.1), рассмотренного в приложении. Для базиса из СКС выполняется условие полноты:

$$
\frac{2 S+1}{4 \pi} \int|\theta, \varphi\rangle\langle\theta, \varphi| \sin \theta d \theta d \varphi=1 .
$$

Однако он не является ортогональным:

$$
\left|\left\langle\theta, \varphi \mid \theta^{\prime}, \varphi^{\prime}\right\rangle\right|^{2}=\cos ^{4 S} \frac{\Theta}{2}, \quad \cos \Theta=\cos \theta \cos \theta^{\prime}+\sin \theta \sin \theta^{\prime} \cos \left(\varphi-\varphi^{\prime}\right) .
$$

Возьмем систему СКC в качестве измерительного базиса в (14). Выполнив POVM-измерение, которое сводится к умножению на СKC и вычислению следа, находим классическую функцию плотности вероятности распределения значений углов:

$$
\begin{aligned}
(4 \pi)^{2} P_{12}\left(\theta_{1}, \varphi_{1}, \theta_{2}, \varphi_{2} ; t\right) & =d_{1} d_{2} \operatorname{Tr}\left\{\left|\theta_{1}, \varphi_{1}\right\rangle\left\langle\theta_{1}, \varphi_{1}|\otimes| \theta_{2}, \varphi_{2}\right\rangle\left\langle\theta_{2}, \varphi_{2}\right| \hat{\rho}(t)\right\}= \\
& =d_{1} d_{2}\left\langle\theta_{1}, \varphi_{1}, \theta_{2}, \varphi_{2}|\hat{\rho}(t)| \theta_{1}, \varphi_{1}, \theta_{2}, \varphi_{2}\right\rangle .
\end{aligned}
$$

Используя свойства СКС

$$
\begin{aligned}
\left\langle\theta_{j}, \varphi_{j}\left|\widehat{S}_{j \pm}\right| \theta_{j}, \varphi_{j}\right\rangle & =S_{j \pm}=S_{j} \sin \theta_{j} e^{ \pm i \varphi_{j}} \\
\left\langle\theta_{j}, \varphi_{j}\left|e^{ \pm i \tau \widehat{S}_{j z}}\right| \theta_{j}, \varphi_{j}\right\rangle & =\left(\cos \frac{\tau}{2} \mp i \cos \theta_{j} \sin \frac{\tau}{2}\right)^{2 S_{j}} \equiv \xi_{j \mp}(t),
\end{aligned}
$$


получаем для функции (29)

$$
P_{12}\left(\theta_{1}, \varphi_{1}, \theta_{2}, \varphi_{2} ; t\right)=\frac{1}{(4 \pi)^{2}}\left\{1+\frac{\beta}{2}\left[S_{1+} \xi_{2+}(t)+S_{1-} \xi_{2-}(t)+S_{2+} \xi_{1+}(t)+S_{2-} \xi_{1-}(t)\right]\right\} .
$$

Дальнейшие вычисления повторяют вычисления для классических моментов, приведенные в приложении. Выполнив интегрирование по углам, находим для редуцированных распределений

$$
P_{1}\left(\theta_{1}, \varphi_{1} ; t\right)=\frac{1}{4 \pi}\left\{1+\beta S_{1 x} g_{2}(t)\right\}, \quad P_{2}\left(\theta_{2}, \varphi_{2} ; t\right)=\frac{1}{4 \pi}\left\{1+\beta S_{2 x} g_{1}(t)\right\}
$$

и для взаимной информации (П.3) в высокотемпературном приближении

$$
J_{\mathrm{BB}}\left(P_{12}\right)=\left(S_{1}\right)^{2} b\left[f_{2}(t)-g_{2}^{2}(t)\right]+\left(S_{2}\right)^{2} b\left[f_{1}(t)-g_{1}^{2}(t)\right],
$$

где

$$
\begin{aligned}
f_{j}(t) & =\frac{1}{2} \int_{-1}^{+1} \xi_{j+}(t) \xi_{j-}(t) d \cos \theta_{j}= \\
& =\sum_{n=0}^{2 S_{j}}\left(\begin{array}{c}
2 S_{j} \\
n
\end{array}\right)\left(\cos \frac{\tau}{2}\right)^{4 S_{j}-2 n}\left(\sin \frac{\tau}{2}\right)^{2 n} \frac{1}{2 n+1}= \\
& =\sum_{n=0}^{2 S_{j}}\left(\begin{array}{c}
2 S_{j} \\
n
\end{array}\right) \frac{(2 n) ! !}{(2 n+1) ! !}(-1)^{n}\left(\sin \frac{\tau}{2}\right)^{2 n} .
\end{aligned}
$$

Эволюция во времени взаимной информации $J_{\mathrm{BB}}\left(P_{12}\right)(31)$, полученной после POVM-измерений с базисом CKC, показана на рис. 1. При малых временах $\tau=$ $t J / S \ll 1$ получаем $J_{\mathrm{BB}}\left(P_{12}\right) \approx J_{\mathrm{c}}\left(P_{\mathrm{c}}\right)$, где $J_{\mathrm{c}}\left(P_{\mathrm{c}}\right)$ определена формулой $(13)$. На больших временах кривая зависимости $J_{\mathrm{BB}}\left(P_{12}\right)$ отходит вниз. Обусловлено это расплыванием пакета из состояний с разными проекциями $S_{z}$ в $\mathrm{CKC}$ (с разными значениями фазового множителя $\left.e^{i \tau S_{z}}\right)$. Тем не менее при $t=T$ пакет соберется, поскольку временна́я зависимость (6) является периодической.

Если теперь из полной корреляции $I(\hat{\rho})(11)$ вычесть найденную классическую часть корреляций (31), то разница даст квантовую часть корреляций:

$$
\begin{aligned}
Q_{\mathrm{BB}} & =I(\hat{\rho})-J_{\mathrm{BB}}\left(P_{12}\right)= \\
& =\left(S_{1}\right)^{2} b\left[1-f_{2}(t)\right]+\left(S_{2}\right)^{2} b\left[1-f_{1}(t)\right]+S_{1} b\left[1-g_{2}^{2}(t)\right]+S_{2} b\left[1-g_{1}^{2}(t)\right] .
\end{aligned}
$$

В рассмотренном выше случае измерения выполнялись симметричным образом на обоих спинах. Другую меру классических корреляций можно получить, проводя POVM-измерение только по одному из спинов, например по второму спину. В этом случае вместо (31) находим

$$
J_{\mathrm{B} 2}=S_{1}\left(S_{1}+1\right) b\left[f_{2}(t)-g_{2}^{2}(t)\right]+\left(S_{2}\right)^{2} b\left[1-g_{1}^{2}(t)\right],
$$

откуда для квантовой части получаем

$$
Q_{\mathrm{B} 2}=I(\hat{\rho})-J_{\mathrm{B} 2}=S_{1}\left(S_{1}+1\right) b\left[1-f_{2}(t)\right]+S_{2} b\left[1-g_{1}^{2}(t)\right] .
$$

Отметим, что в системах с непрерывным спектром ранее была введена мера, названная гауссовским квантовым дискордом, для получения которой используются POVM-измерения с базисом из полевых (бозонных) когерентных состояний [37], [38]. 


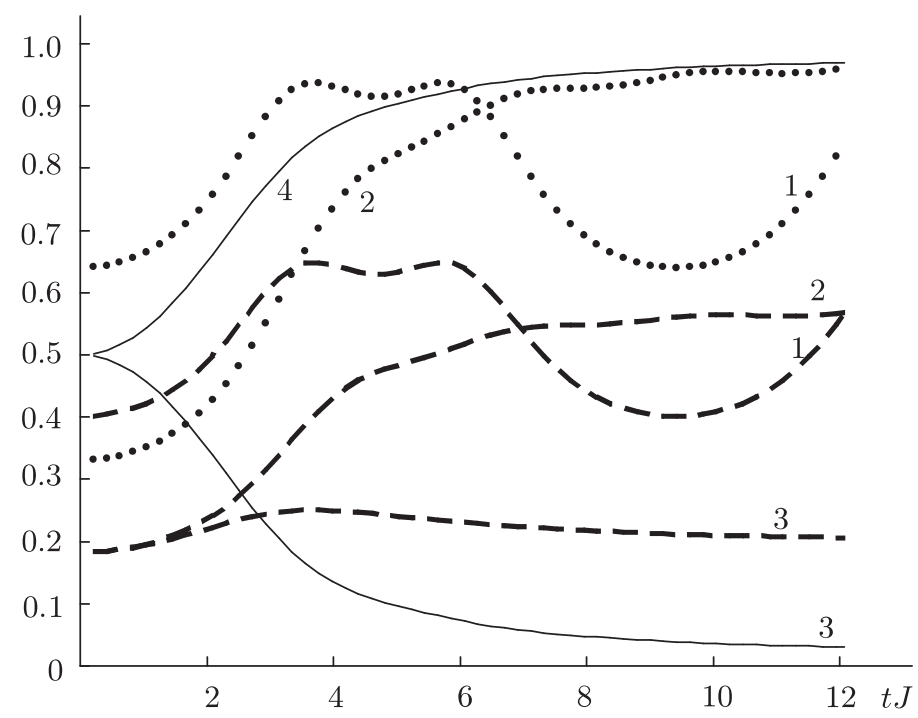

Рис. 2. Эволюция во времени квантовой части корреляций для разных пар спинов, обозначенных цифрами у кривых: $S_{1}=S_{2}=3 / 2(1) ; S_{1}=S_{2}=$ $9 / 2(2) ; S_{1}=1 / 2, S_{2}=9 / 2(3) ; S_{1}=9 / 2, S_{2}=1 / 2(4)$. Результаты ортогональных измерений по первому спину $D_{\mathrm{N}} / I(\hat{\rho})$ и $Q_{\mathrm{N}}^{(S 1)} / I(\hat{\rho})$ показаны сплошными линиями. Результаты неортогональных измерений показаны пунктирными линиями при измерении по обоим спинам $Q_{\mathrm{BB}} / I(\hat{\rho})$ и штриховыми линиями - при измерении только по второму спину $Q_{\mathrm{B} 2} / I(\hat{\rho})$.

\section{5. ОБСУЖДЕНИЕ}

Временна́я зависимость долей квантовых корреляций, полученных в результате разных способов измерений, показана на рис. 2 в виде отношений $Q_{\mathrm{BB}} / I(\hat{\rho})$, $Q_{\mathrm{B} 2} / I(\hat{\rho}), Q_{\mathrm{N}}^{(S 1)} / I(\hat{\rho})$ и $D_{\mathrm{N}} / I(\hat{\rho})$ при разных значениях $S_{1}$ и $S_{2}$. На малых временах $\tau=t J / S_{2} \ll 1$ по формулам (33), (35) и (12) находим для результатов POVMизмерений по базису из СKC

$$
\frac{Q_{\mathrm{BB}}}{I(\hat{\rho})} \approx \frac{S_{1}+S_{2}+1}{\left(S_{1}+1\right)\left(S_{2}+1\right)}, \quad \frac{Q_{\mathrm{B} 2}}{I(\hat{\rho})} \approx \frac{1}{S_{2}+1},
$$

что согласуется с данными, приведенными на рис. 2. Из результатов (23) и (26), полученных после проецирования на ортогональный базис (25), на малых временах следует равенство классического и квантового вкладов: $C_{\mathrm{N}}^{(S 1)}=Q_{\mathrm{N}}^{(S 1)}=I(\hat{\rho}) / 2$. При $S_{1}=S_{2}$ это равенство сохраняется для всех времен. Ранее равенство вкладов было получено в работе [13] в частном случае $S_{1}=S_{2}=1 / 2$.

Для выяснения причин различий двух способов измерений рассмотрим матрицу плотности (6) на малых временах

$$
\hat{\rho}(t) \sim \frac{1}{Z}\left[1+\beta\left(\widehat{S}_{1 x}+\widehat{S}_{2 x}\right)+\frac{\beta t J}{S_{2}}\left(\widehat{S}_{1 y} \widehat{S}_{2 z}+\widehat{S}_{2 y} \widehat{S}_{1 z}\right)\right] .
$$


После ортогонального измерения (16) с проекторами (25) вклад от слагаемого $\widehat{S}_{1 y} \widehat{S}_{2 z}$ равен нулю, поэтому остающаяся после измерения классическая часть корреляций составит половину полных корреляций при любом значении спина. При POVM-измерении состояния (37) по второму (большому) спину оба слагаемых дают вклады в классическую часть корреляций (34) (или в (31) при POVM-измерениях состояния (37) по обоим спинам). Теряются только квантовые флуктуации при вычислении среднего от квадрата оператора спиновой компоненты: $S_{j}^{2} / 3$ вместо $S_{j}\left(S_{j}+1\right) / 3$. При $S_{j} \rightarrow \infty$ эти различия, обусловленные флуктуациями, исчезают. Тем самым мы получаем, что при увеличении спина растет доля классических корреляций и соответственно уменьшается доля квантовых корреляций, которая стремится к нулю в пределе $S_{j} \rightarrow \infty$.

Вывод о том, что максимум вклада классических корреляций достигается при POVM-измерениях, сам по себе не нов и согласуется с общей теорией [7]. Однако для рассматриваемого чаще других случая $S_{1}=S_{2}=1 / 2$ переход от ортогональных измерений фон Неймана к POVM-измерениям приводит к незначительным по величине уточнениям [7]. Разница результатов для классической взаимной информации при двух способах измерений возрастает в системе из кубита и кутрита [39]. Мы получили подобный результат для системы двух больших спинов в состоянии с матрицей плотности (37). По крайней мере для этого состояния методы оценки, основанные на ортогональных измерениях, могут исказить реальное соотношение квантовых и классических корреляций.

Перейдем к анализу эволюции квантовых корреляций на больших временах. При $t=T$ корреляции в рассматриваемой квантовой системе исчезают, поскольку $I(\hat{\rho})=$ 0 . При $t \approx T / 2$ коррелированность максимальна, как это видно на графиках взаимной информации на рис. 1. Каковы при этом доли квантовых и классических корреляций, зависит от способа измерения (см. рис. 2). Результаты (23) и (26), полученные после проецирования на ортогональный базис (25), приводят при $S_{1} \neq S_{2}$ к периодической временно́й зависимости долей классических и квантовых корреляций. Эти доли равны между собой при малых временах, но достигают при $\sin ^{2}(\tau / 2)=1$ разных значений:

$$
\frac{C_{\mathrm{N}}^{(S 1)}}{I(\hat{\rho})} \approx \frac{S_{2}\left(S_{2}+1\right)}{S_{1}\left(S_{1}+1\right)+S_{2}\left(S_{2}+1\right)}, \quad \frac{Q_{\mathrm{N}}^{(S 1)}}{I(\hat{\rho})} \approx \frac{S_{1}\left(S_{1}+1\right)}{S_{1}\left(S_{1}+1\right)+S_{2}\left(S_{2}+1\right)} .
$$

Такие зависимости показаны на рис. 2 при $S_{1}=1 / 2, S_{2}=9 / 2$ и $S_{1}=9 / 2, S_{2}=$ $1 / 2$. Отношение $Q_{\mathrm{N}}^{(S 1)} / I(\hat{\rho})$ согласно $(11),(23),(26)$ со временем или уменьшается (при $S_{1}<S_{2}$ ), или увеличивается (при $S_{1}>S_{2}$ ) (в последнем случае $S_{2}$ заменяем на $S_{1}$ при масштабировании параметров $J$ и $\tau$ ). С другой стороны, после POVM-измерений мы получаем из (31) и (34) при $\sin ^{2}(\tau / 2)=1$ (в ряду (32) остается только один член $\left.f_{j}(t)=1 /\left(4 S_{j}+1\right)\right)$

$$
\frac{J_{\mathrm{B} 2}}{I(\hat{\rho})} \approx \frac{S_{1}\left(S_{1}+1\right) /\left(4 S_{2}+1\right)+S_{2}^{2}}{S_{1}\left(S_{1}+1\right)+S_{2}\left(S_{2}+1\right)}, \quad \frac{J_{\mathrm{BB}}}{I(\hat{\rho})} \approx \frac{S_{1}^{2} /\left(4 S_{2}+1\right)+S_{2}^{2} /\left(4 S_{1}+1\right)}{S_{1}\left(S_{1}+1\right)+S_{2}\left(S_{2}+1\right)} .
$$

Далее из (33) и (35) мы находим оценки при $S_{1}=S_{2}=S \gg 1$ :

$$
\frac{Q_{\mathrm{BB}}}{I(\hat{\rho})} \approx 1-\frac{1}{4 S} \quad \text { и } \quad \frac{Q_{\mathrm{B} 2}}{I(\hat{\rho})} \approx \frac{1}{2}\left(1+\frac{3}{4 S}\right) .
$$


На основании рис. 2 и приведенных формул заключаем, что при $t \sim T / 2$ ортогональное измерение позволяет добиться меньшего значения для доли квантовых корреляций, чем POVM-измерение с базисом из CKC, т. е. получаем соотношение результатов, противоположное случаю малых времен. Для объяснения этого выпишем матрицу плотности при $t=T / 2(\tau=\pi)$ :

$$
\hat{\rho}\left(\frac{T}{2}\right)=\frac{1}{Z}\left\{1+\frac{\beta}{2}\left[\widehat{S}_{1+} e^{-i \pi \widehat{S}_{2 z}}+\widehat{S}_{1-} e^{i \pi \widehat{S}_{2 z}}+\widehat{S}_{2+} e^{-i \pi \widehat{S}_{1 z}}+\widehat{S}_{2-} e^{i \pi \widehat{S}_{1 z}}\right]\right\} .
$$

При изменении значения спиновых проекций на единицу функция $e^{i \pi S_{j z}}$ меняет знак. Соответствующие слагаемые в (41) имеют вид $\beta\left[\widehat{S}_{1 x}(-1)^{m_{2}}+\widehat{S}_{2 x}(-1)^{m_{1}}\right]$ для целочисленных спинов и $\beta\left[\widehat{S}_{1 y}(-1)^{m_{2}-1 / 2}+\widehat{S}_{2 y}(-1)^{m_{1}-1 / 2}\right]$ для полуцелых. При ортогональных измерениях с проекторами (25) по одному из спинов (первому) вклад дает одно слагаемое. При этом, в отличие от случая малых времен, величина вклада этого слагаемого изменится. Например, при $S_{1}<S_{2}$ остается вклад "маленькое поле от маленького спина поворачивает большой спин", тогда как вклад "большой спин поворачивает малый" теряется. Мы получаем выражения (38) для долей классических и квантовых корреляций. Результат проецирования (29) состояния (41) на CKC при POVM-измерении сложнее. CKC (27) образовано суммой состояний (5) с разными проекциями спина на ось $z$, которые дадут в (29) и (30) знакопеременные ряды с суммами:

$$
\xi_{j \mp}\left(\frac{T}{2}\right)=\left[\cos \theta_{j}\right]^{2 S_{j}} .
$$

В итоге находим после POVM-измерений результаты (39), уступающие по величине результату ортогональных измерений (38).

Мы выполнили POVM-измерения с базисом из CKC как на одном, так и на двух спинах. Доля квантовых корреляций в первом случае меньше. С другой стороны, при ортогональных измерениях состояния (37) по одному или по двум спинам получаем одни и те же значения классической и, следовательно, квантовой корреляций. Действительно, если после ортогонального измерения по первому спину произвести ортогональное проецирование второго спина на собственные состояния оператора $\widehat{S}_{2 y}$, то дополнительных потерь корреляций не произойдет. Подобное наблюдение о совпадении результатов измерений по одному и двум кубитам сделано в работе [39]. Как измерять - еще не решенный окончательно вопрос [7], [39], [40]. Так, по мнению Ло [40], при измерении на одном спине получается мера квазиклассической корреляции, которая включает в себя часть квантовых корреляций.

\section{6. ЗАКЛЮЧЕНИЕ}

Итак, наблюдаемые методом ЯМР на малых временах корреляции (3) для нашей модели принимают вид (37). Для двух спинов $S_{1}=S_{2}=1 / 2$ эти корреляции делятся поровну на квантовую и классическую части, согласно результатам ортогональных измерений. При увеличении спина растет доля классических корреляций и уменьшается соответственно доля квантовых корреляций, которая стремится к нулю в пределе $S \rightarrow \infty$. Для получения такого результата мы перешли от ортогональных измерений к POVM-измерениям с базисом из СКС. С ростом времени 
линейная временна́я зависимость сменяется зависимостью в виде суммы экспоненциальных функций с разными периодами. По прошествии времени $T=2 \pi S / J$, равного наибольшему общему кратному этих периодов при $S_{1}=S_{2}=S$, корреляции снова обращаются в нуль. На временах, близких к половине периода, наблюдается наибольшая степень коррелированности двух спинов, характеризуемая максимумом взаимной информации. В этой области относительная доля квантовых корреляций возрастает по сравнению с областью малых времен. Поскольку при таких временах фазовые множители соседних состояний с проекциями, отличающимися на единицу, имеют разные значения, то POVM-измерение с базисом из CKC становится слишком грубым измерителем. Дело в том, что в СКС разброс проекций $m$ имеет порядок величины $\sqrt{S} \gg 1$ [31]. Ортогональные измерения при проецировании на отдельные состояния с определенными значениями $m$ позволяют извлечь больше классических корреляций. Тем не менее квантовые корреляции составляют заметную часть, равную половине всех корреляций при $S_{1}=S_{2}$. При $S_{1}<S_{2}$ их доля уменьшается в $\left(S_{1} / S_{2}\right)^{2}$ раз, если измерять по первому спину.

ПРИЛОЖЕНИЕ

\section{Взаимная информация двух классических моментов}

Рассмотрим систему из двух классических магнитных моментов. Будем описывать их состояния полярными $\theta$ и азимутальными $\varphi$ углами на единичной сфере (сфере Блоха), через которые могут быть выражены проекции классического момента $\vec{S}$ на координатные оси:

$$
S_{z}=S \cos \theta, \quad S_{y}=S \sin \theta \sin \varphi, \quad S_{x}=S \sin \theta \cos \varphi .
$$

Вместо матрицы плотности $\hat{\rho}(t)(6)$ состояния ансамбля описывается теперь функцией плотности распределения вероятности значений углов на сфере Блоха:

$$
\begin{aligned}
& P_{\mathrm{c}}\left(\theta_{1}, \varphi_{1}, \theta_{2}, \varphi_{2} ; t\right)= \\
& \quad=\frac{1}{(4 \pi)^{2}}\left\{1+\frac{\beta}{2}\left[S_{1+} e^{-i \tau S_{2 z}}+S_{1-} e^{i \tau S_{2 z}}+S_{2+} e^{-i \tau S_{1 z}}+S_{2-} e^{i \tau S_{1 z}}\right]\right\},
\end{aligned}
$$

где

$$
S_{j z}=S_{j} \cos \theta_{j}, \quad S_{j \pm}=S_{j} \sin \theta_{j} e^{ \pm i \varphi_{j}} .
$$

Классическая взаимная информация имеет вид

$$
J_{\mathrm{c}}\left(P_{\mathrm{c}}\right)=S_{\mathrm{Sh}}\left(P_{\mathrm{c} 1}\right)+S_{\mathrm{Sh}}\left(P_{\mathrm{c} 2}\right)-S_{\mathrm{Sh}}\left(P_{\mathrm{c}}\right),
$$

где

$$
\begin{aligned}
& S_{\mathrm{Sh}}\left(P_{\mathrm{c}}\right)= \\
& \quad=-\iint P_{\mathrm{c}}\left(\theta_{1}, \varphi_{1}, \theta_{2}, \varphi_{2} ; t\right) \log _{2} P_{\mathrm{c}}\left(\theta_{1}, \varphi_{1}, \theta_{2}, \varphi_{2} ; t\right) \sin \theta_{2} d \theta_{2} d \varphi_{2} \sin \theta_{1} d \theta_{1} d \varphi_{1}
\end{aligned}
$$

- энтропия Шеннона. Вместо взятия следа надо вычислять интеграл по сфере Блоха. Редуцированная плотность распределения для первого момента (для второго 
вычисляется аналогично)

$$
\begin{aligned}
P_{\mathrm{c} 1}\left(\theta_{1}, \varphi_{1} ; t\right) & =\int P_{\mathrm{c}}\left(\theta_{1}, \varphi_{1}, \theta_{2}, \varphi_{2} ; t\right) \sin \theta_{2} d \theta_{2} d \varphi_{2}= \\
& =\frac{1}{4 \pi}\left\{1+\beta S_{1 x} g_{\mathrm{c} 2}(t)\right\}, \\
g_{\mathrm{c} j}(t) & =\frac{1}{2} \int_{-1}^{+1} e^{ \pm i \tau S_{j} \cos \theta_{j}} d \cos \theta_{j}=\frac{\sin \tau S_{j}}{\tau S_{j}} .
\end{aligned}
$$

Находим для классической взаимной информации (П.3) в высокотемпературном приближении следующее выражение:

$$
J_{\mathrm{c}}\left(P_{\mathrm{c}}\right)=\left(S_{1}\right)^{2} b\left[1-g_{\mathrm{c} 2}^{2}(t)\right]+\left(S_{2}\right)^{2} b\left[1-g_{\mathrm{c} 1}^{2}(t)\right] .
$$

\section{Список литературы}

[1] J. A. Jones, Prog. NMR Spectrosc., 59:2 (2011), 91-120, arXiv: 1011.1382.

[2] R. L. Auccaise, C. Céleri, D. O. Soares-Pinto, E. R. deAzevedo, J. Maziero, A. M. Souza, T. J. Bonagamba, R. S. Sarthour, I. S. Oliveira, R. M. Serra, Phys. Rev. Lett., 107:14 (2011), 140403, 5 pp.

[3] G. Passante, O. Moussa, R. Laflamme, Phys. Rev. A, 85:3 (2012), 032325, 5 pp.

[4] G. Passante, O. Moussa, C. A. Ryan, R. Laflamme, Phys. Rev. Lett., 103:25 (2009), 250501, $4 \mathrm{pp}$.

[5] A. Datta, A. Shaji, C. M. Caves, Phys. Rev. Lett., 100:5 (2008), 050502, 4 pp.

[6] A. F. Fahmy, R. Marx, W. Bermel, S. J. Glasser, Phys. Rev. A, 78:2 (2008), 022317, 10 pp.

[7] K. Modi, A. Brodutch, H. Cable, T. Paterek, V. Vedral, Rev. Modern Phys., 84:4 (2012), $1655-1707$.

[8] А. Абрагам, М. Гольдман, Ядерный магнетизм: порядок и беспорядок, т. 1, Мир, М., 1984.

[9] I. G. Powles, P. Mansfield, Phys. Lett., 2:2 (1962), 58-59.

[10] J. Jeener, P. Broekaert, Phys. Rev., 157:2 (1967), 232-240.

[11] B. C. Sanders, Phys. Rev. A, 40:5 (1989), 2417-2427.

[12] G. J. Milburn, R. Laflamme, B. C. Sanders, E. Knill, Phys. Rev. A, 65:3 (2002), 032316, $10 \mathrm{pp}$.

[13] E. I. Kuznetsova, A. I. Zenchuk, Phys. Lett. A, 376:10-11 (2012), 1029-1034.

[14] X. Peng, D. Suter, D. A. Lidar, J. Phys. B, 44:15 (2011), 154003, 6 pp.

[15] J. Baugh, A. Kleinhammes, D. Han, Q. Wang, Y. Wu, Science, 294:5546 (2001), 1505-1507.

[16] D. Gottesman, Lect. Notes Comp. Sci., 1509 (1999), 302-313, arXiv: quant-ph/9802007.

[17] A. Muthukrishnan, C. R. Stroud (Jr.), Phys. Rev. A, 62:5 (2000), 052309, 8 pp.

[18] В. Е. Зобов, А. С. Ермилов, ЖКЭТФ, 141:6 (2012), 1060-1070.

[19] A. Miranowicz, P. Horodecki, R. W. Chhajlany, J. Tuziemski, J. Sperling, Phys. Rev. A, 86:4 (2012), 042123, 10 pp.

[20] E. B. Fel'dman, A. I. Zenchuk, Phys. Rev. A, 86:1 (2012), 012303, 9 pp.

[21] E. B. Fel'dman, E. I. Kuznetsova, M. A. Yurishchev, J. Phys. A, 45:47 (2012), 475304, 11 pp.

[22] M. Daoud, R. Ahl Laamara, Internat. J. Quantum Inf., 10:5 (2012), 1250060, 23 pp., arXiv: 1210.8313 .

[23] R. Rossignoli, J. M. Matera, N. Canosa, Phys. Rev. A, 86:2 (2012), 022104, 7 pp.

[24] E. Ghitambar, Phys. Rev. A, 86:3 (2012), 032110, 9 pp. 
[25] S. Vinjanampathy, A. R. P. Rau, J. Phys. A, 45:9 (2012), 095303, 10 pp.

[26] Шунь-Лун Ло, Шуан-Шуан Фу, ТМФ, 171:3 (2012), 519-528.

[27] A. S. M. Hassan, B. Lari, P. S. Joag, Phys. Rev. A, 85:2 (2012), 024302, 6 pp., arXiv: 1010.1920.

[28] T. Tufarelli, T. MacLean, D. Girolami, R. Vasile, G. Adesso, J. Phys. A, 46:27 (2013), 275308, 15 pp., arXiv: 1301.3526.

[29] Д. Прескилл, Квантовая информация и квантовые вычисления, т. 1, РХД, М.-Ижевск, 2008.

[30] F. T. Arrechi, E. Courtens, R. Gilmore, H. Thomas, Phys. Rev. A, 6:6 (1972), 2211-2237.

[31] J. Kofler, C. Brukner, Phys. Rev. Lett., 101:9 (2008), 090403, 4 pp., arXiv: 1009.2654.

[32] M. Piani, Phys. Rev. A, 86:3 (2012), 034101, 3 pp.

[33] S. Rana, P. Parashar, Phys. Rev. A, 86:3 (2012), 030302, 4 pp.

[34] A. Peres, Phys. Rev. A, $46: 7$ (1992), 4413-4414.

[35] G.S. Agarwal, Phys. Rev. A, 47:6 (1993), 4608-4615.

[36] A. Polkovnikov, Ann. Phys., 325:8 (2010), 1790-1852, arXiv: 0905.3384.

[37] G. Adesso, A. Datta, Phys. Rev. Lett., 105:3 (2010), 030501, 4 pp.

[38] P. Giorda, M. G. A. Paris, Phys. Rev. Lett., 105:2 (2010), 020503, 4 pp.

[39] S. Wu, U. V. Poulsen, K. Mølmer, Phys. Rev. A, 80:3 (2009), 032319, 11 pp.

[40] S. Luo, Phys. Rev. A, 77:2 (2008), 022301, 5 pp.

Поступила в редакцию 2.02.2013,

после доработки 2.04.2013 